\title{
Protée
}

\section{Du pictural au photographique. Une rencontre re-visitée}

\section{Marie-Josée Pinard}

Volume 28, numéro 3, 2000

Mélancolie entre les arts

URI : https://id.erudit.org/iderudit/030601ar

DOI : https://doi.org/10.7202/030601ar

Aller au sommaire du numéro

Éditeur(s)

Département des arts et lettres - Université du Québec à Chicoutimi

ISSN

0300-3523 (imprimé)

1708-2307 (numérique)

Découvrir la revue

Citer cet article

Pinard, M.-J. (2000). Du pictural au photographique. Une rencontre re-visitée. Protée, 28(3), 23-29. https://doi.org/10.7202/030601ar

\section{Résumé de l'article}

De cette rencontre entre le pictural et le photographique advenue au XIX ${ }^{e}$ siècle, la littérature ne semble avoir retenu que le «portrait » défait du pictural : une défection, à la fois proposée comme un « manque qui s’inscrit » dans l'ordre de sa performance mimétique et comme une symbolique de son "re-trait » de l'autre vers soi. De l'avènement de cette prolongation mécanique du geste de l'artiste, on a décrié haut et fort à la mort de la peinture ! Que cela soit par le manque de transférabilité de son vocabulaire analytique ou par l'insuffisance de son rendu mimétique, toute la problématique de cette rencontre s'est échafaudée autour de l'adéquation platonicienne du rapport au même. " Autre " est cette lecture que nous proposons - lecture différentielle de cette rencontre " originaire » qui, en utilisant comme élément vectoriel la portabilité, s'inscrit davantage comme une des étapes médiatiques d'un monde qui se veut de plus en plus idéel. 


\section{DU PICTURALAU PHOTOGRAPHIQUE U NE REN CONTRE RE-VISITÉE}

MARIE-JO SÉE PINARD

- Qu'est-ce proprement que l'aura? Une trame singulière d'espace et de temps: unique apparition d'un lointain, si proche soit-il. Reposant l'été, à l'heure de midi, suivre à l'horizon la ligne d'une chaîne de montagnes ou une branche qui jette son ombre sur celui qui la contemple, jusqu'à ce que l'instant ou l'heure ait part à leur manifestation, - c'est respirer l'aura de ces montagnes, de cette branche. Mais en réalité rapprocher les choses de soi, surtout des masses, c'est chez les hommes d'aujourd'hui une disposition exactement aussi passionnée que leur tendance à maîtriser l'unicité de tout donné en accueillant la reproduction de ce donné. De jour en jour le besoin s'impose davantage de posséder de l'objet la plus grande proximité possible, dans l'image et surtout dans la reproduction. [...] [C'est l'auteur qui souligne]

Walter Benjamin (1931)

Le paradigme traditionnel d'une mise en relation de la photographie d'avant 1950 et de la peinture se conjugue sous différents aspects. D'une part, d'une mise en parallèle de leurs performances mimétiques respectives s'est dressé l'échafaud que le photographique destinait au pictural - cette fameuse mort de la peinture! De cette rencontre mortifère, la peinture s'est alors tournée vers d'autres cieux - vers l'autoréférentialité. D'autre part, la venue de ce nouveau médium a notamment bousculé la notion d'originalité de l'œuvre, celle de la vocation artistique, du corpus, de la stylistique et de la signature; elle a donc porté à sa limite la transférabilité du vocabulaire analytique pictural à celui de l'image photographique. Que ce soit par une approche différentielle du processus de "création» impliqué dans la réalisation de l'objet, du making pictural au taking photographique, ou du processus analytique formel versus utilitaire (par usages), une dimension analytique de ce rapprochement du photographique et du pictural semble avoir été occultée dans la littérature: celle de la portabilité.

La portabilité s'entend comme le "caractère d'un appareil, d'un matériel ou d'un programme portable» ${ }^{1}$. Inspiration faite d'un rapprochement étymologique entre les substantifs portabilité et portrait, il est proposé dans cet article de mettre en 
lumière comment la portabilité se présentait comme une nécessité intrinsèque du pictural au photographique, c'est-à-dire comment la peinture portait en son sein l'avènement du médium photographique, considéré de ce fait comme une autre étape médiatique vers un monde de plus en plus idéel.

Compte tenu de l'ampleur du projet, il est proposé d'esquisser ici, pour filer la métaphore médiatique, les raisons qui justifient la pertinence du choix du portrait comme véhicule analytique de ce point de rencontre intermédial appréhendé sous l'«optique» de la portabilité.

\section{PORTABILITÉ ET PORTRAIT:}

\section{RAPPROCHEMENTS ÉTYMOLOGIQUES}

Les vocables "portabilité» et "portrait», malgré leur consonance séduisante et quasi trompeuse, ne partagent pas la même racine étymologique. «Portabilité» se rattache au verbe latin portare et «portrait» tire son origine du verbe trahere en latin classique ou de tragere en latin vulgaire.

\section{PORTABILITÉ}

Le substantif portabilité est dérivé du verbe porter, apparu au Xe siècle et qui, selon le Dictionnaire étymologique et historique de la langue française ${ }^{2}$, est issu du latin vulgaire portare; portare signifiait alors «transporter, porter", au sens figuré de "porter secours, nouvelles, etc.». Dans l'ancien français, "porter" s'entendait aussi dans le sens de "porter un enfant", de "produire (en parlant de fruits)", de "porter sur soi ou avec soi», ainsi qu'au sens de «déplacer, transporter et apporter».

Selon le Dictionnaire étymologique de la langue latine. Histoire de mots ${ }^{3}$, le verbe portare aurait remplacé, à la fin de l'époque impériale, le verbe latin ferre (latin classique). Il convient de préciser ici certaines significations engagées par l'apposition à ce verbe des préfixes " pro- et re-» pour profero "produire au dehors", "publier", et refero "reproduire, représenter et répéter». La "portabilité» engagerait ainsi par son historique, en sus de sa définition de «porter avec soi», une connotation à la fois de "production/ publication» et de «re-production/re-présentation». Elle contiendrait donc en son sens originaire déjà une référence médiatique.

\section{PORTRAIT}

Le terme portrait est le participe passé substantivé du verbe portraire, "dessiner, représenter», qui est apparu au XIIe siècle et sorti d'usage au XVIIIe siècle; il est composé de pour et de traire au sens de "dessiner». Le verbe traire, apparu au XI siècle, provient, quant à lui, du latin classique trahere, employé en ancien français au sens de «tirer, attirer » et de «sup-porter» ou du latin vulgaire tragere, «tirer, traîner». Traîner, verbe qui apparut au début du XIIe siècle et que l'on utilisait dans le sens de «tirer derrière soi» - $d^{\prime}$ " emmener partout avec soi » ${ }^{4}$, et qui anticipait ainsi d'ores et déjà la nécessité future du vocable portabilité, est, selon le Dictionnaire étymologique $d u$ français $^{5}$, issu du latin populaire traginare, dérivé lui-même de tragere ou du classique trahere. Force est de constater que traîner, terme annonciateur de la «portabilité» et traire (pour-traire) partagent la même origine étymologique, tragere/trahere dans le sens d'«emporter avec soi", «tirer».

Le portrait portait donc en son sein la portabilité. Qui plus est, le Dictionnaire $d u$ français prête les divers usages suivants au verbe «tirer»:

1.Tracer. Tirer un trait, une ligne. $\triangleright$ Tirer un plan, le dessiner. - Par ext., fig. Tirer des plans: élaborer, mûrir des projets. 2. Imprimer. Tirer un ouvrage sur papier bible. $\triangleright$ v. intr. Être reproduit, imprimé, gravé. Journal qui tire à un million d'exemplaires. $\triangleright$ PHOTO Faire un tirage. - Loc. fam. Tirer le portrait à quelqu'un, faire son portrait, sa photographie. 6

Tout l'historique médiatique qui va du dessin à la peinture, à l'imprimerie, à la gravure et à la photographie est représenté par la déclinaison de ce verbe au fil du temps pour se terminer sur cette locution familière "Tirer le portrait à quelqu'un» qui ne fait que renforcer l'importance de cette trilogie peinture, photographie et portabilité du portrait. Le portrait porte ainsi, non seulement en son sein la 
portabilité, mais il se confond quasi ontologiquement à son support médiatique tout au fil du temps.

Dans cet esprit, il est intéressant de se rappeler la vision qu'Abraham Bosse, ce graveur français du XVII siècle, avait de la "portraiture» comme étant «un mot général comprenant et la Peinture et la Gravure», et "pour lui, "portrait" signifiait la même chose que "tableau" "7. Il y avait donc congruence du support et du "genre», tout en dénonçant le cloisonnement engendré par le caractère restrictif de cette nomenclature picturale.

\section{IMPORTANCE HISTORIQUE DU PORTRAIT}

Que le portrait porte en lui la portabilité et que sa déclinaison historique reflète à son tour tout le "portrait» médiatique est une condition nécessaire mais non suffisante de son utilisation comme véhicule analytique de la mise en relation de la peinture et de la photographie sous ce nouvel angle de la portabilité. Il appert essentiel d'établir également l'importance respective soit symbolique/métaphorique, soit métonymique du portrait par rapport au pictural et au photographique.

\section{LA PEINTURE}

La peinture portait en son origine le portrait. Deux passages de l'Histoire naturelle de Pline l'Ancien ont, en effet, établi la doctrine canonique du mythe originaire de la peinture qui se confond avec celui du portrait. Dans son récent ouvrage sur les Théories $d u$ portrait. De la Renaissance aux Lumières, Édouard Pommier rapporte:

$$
\begin{aligned}
& \text { Au chapitre XV du livre XXXV, Pline l'Ancien évoque } \\
& \text { quelques traditions, égyptiennes et grecques, sur ce thème; sans se } \\
& \text { prononcer lui-même clairement, il [Pline] conclut prudemment: } \\
& \text { "tous reconnaissent qu'il [principe de la peinture] a consisté à } \\
& \text { tracer, grâce à des lignes, le contour d'une ombre humaine». }{ }^{8}
\end{aligned}
$$

Voilà donc établie la thèse de la circumductio umbrae, de la circonscription de l'ombre. Il y a déjà à l'origine de la peinture à la fois l'image de l'ombre de l'homme, sa silhouette, et le dessin. Toujours selon Pommier, Pline l'Ancien poursuit ainsiau chapitre CLI:
[...] le potier Butadès, à Corinthe, dut son invention à sa fille, qui était amoureuse d'un jeune homme: celui-ci partant pour l'étranger, elle entoura d'une ligne l'ombre de son visage projetée sur le mur par la lumière d'une lanterne; son père appliqua de l'argile sur l'esquisse, en fit un relief qu'il mit à durcir au feu avec le reste de ses poteries, après l'avoir fait sécher. ${ }^{9}$

À ce discours premier sur la peinture ${ }^{10}$ viennent donc s'ajouter ici, en sus de l'image de l'ombre de l'homme, de sa silhouette, et du dessin, la motivation du geste, l'amour. L'amour, ce "grand maître des inventions» dit André Félibien ${ }^{11}$, incite ainsi à pallier l'absence de l'être aimé, à réduire par le portrait cette distance «temporelle " qui sépare les deux amants mélancoliques. Cette addition connotative permet de dégager comme fonction première du portrait celle de la présentéification de l'absent et provoque ainsi une lecture du portrait comme fétiche, au sens freudien du terme ${ }^{12}$, c'est-à-dire comme matérialité, objetfétiche, et non comme chez Lacan une projection idéelle ${ }^{13}$.

Il existe d'ailleurs nombre d'exemples littéraires pour étoffer (sans jeu de mots) ${ }^{14}$ cette prise en considération du portrait comme objet fétichiste. Je pense ici à La Galeria (1620), ouvre du poète italien Giovanni Battista Marino [1569-1625] où l'on retrouve l'image du poète qui, emporté dans une sorte de délire devant le portrait de la femme aimée,

[...] en vient, dans sa douleur impuissante, à mordre le cadre qui renferme l'image de celle qu'il ne peut embrasser, pour trouver "dans une couleur feinte une douleur vraie». 15

Il y a lieu de mentionner également le portrait de Madame de Clèves dérobé de son écrin par le Prince de Nemours dans le roman de Madame de La Fayette (1634-1693) ou le portrait-talisman de Sylvie dérobé à Des Frans lors de son passage dans les Alpes, dont nous parle Robert Challe (1659-1721) dans Les Illustres Françaises ${ }^{16}$.

C'est également par cette fonction mémoriale, cette présentéification de l'absent, qu'Alberti introduit le portrait dans son traité théorique fondateur de la peinture, le De Pictura (1435): 
Comme l'application qu'il faut mettre à cette étude risque de sembler trop pénible aux jeunes gens, je pense qu'il convient ici de montrer que la peinture mérite pleinement que nous lui consacrions notre travail et notre attention. Elle a en elle une force tout à fait divine qui lui permet non seulement de rendre présent, comme on le dit de l'amitié, ceux qui sont absents, mais aussi de montrer après plusieurs siècles les morts aux vivants, de façon à les faire connaître pour le plus grand plaisir de ceux qui regardent dans la plus grande admiration pour l'artiste. Plutarque rapporte que Cassandre, l'un des généraux d'Alexandre, se mit à trembler de tout son corps en regardant une image dans laquelle il reconnaissait Alexandre qui était déjà mort et voyait en elle la majesté du roi; qu'Agésilas le Lacédémonien, parce qu'il se savait très laid, refusa de laisser son effigie à la postérité et, pour cette raison, ne permit jamais qu'on fit son portrait ou sa statue. C'est donc que les visages des défunts prolongent d'une certaine manière leur vie par la peinture. ${ }^{17}$

Dans le fil de cette référence à Plutarque, Alberti dote le portrait d'une valeur transcendantale autre que celle de l'amour avancée par Pline l'Ancien; de la présentéification de l'absent aimé, on passe ici à la fonction politique du portrait (portrait du roi, d'apparat, etc.). On retrouve d'ailleurs très bien développée chez Paleotti (1522-1597), dans son Discorso intorno alle imagini sacre e profane, cette cohabitation de la matérialité et de la transcendance dans le portrait, c'est-à-dire du réalisme du référent (personne privée) et de l'intemporalité de la fonction (personne publique). Voici ce que l'on peut lire dans la théorie des «deux corps» développée par Paleotti:

Les princes chrétiens, que Dieu a placés à leur rang comme des lois vivantes et des instruments de la justice et sagesse divines pour le gouvernement des peuples, portent deux personnes ensemble: une personne publique et une personne privée. 18

Dans son livre l'Histoire du portrait en cire, Schlosser fait retour sur cette duplication ontologique du portrait en s'inspirant du livre mythique Les Deux Corps du roi ${ }^{19}$ d'Ernst Kantorowicz. Chez Schlosser, la duplication du portrait n'est toutefois pas ontologique, mais reflète plutôt les deux fonctions différentes réservées au corps réel et à son effigie. En effet, [c]ette forme de duplication, où s'exprime un rituel plus subtil, a conditionné le déroulement des cérémonies funéraires des rois de France. Après avoir conduit en cortège le cadavre et sa représentation en cire d'après le naturel, on s'occupait séparément de l'un et de l'autre: au premier exclusivement on réservait le deuil, tandis qu'on destinait à la seconde le grandiose déploiement des fastes princiers. Dans son ouvrage mythique Les Deux Corps du roi [...], Ernst Kantorowicz a démontré de façon éclatante que c'est à juste titre que l'effigie ornée, dans cette duplication des insignes royaux, était nommée représentation [...]. La représentation artificielle et authentiquement ressemblante n'avait aucune fonction de substitution, car l'intention n'était plus de rendre présent le défunt, mais plutôt de montrer concrètement des principes dynastiques et politiques. Avec le sceptre, la couronne, le manteau, les décorations et les autres insignes, le corps fictif s'opposait au corps naturel du roi, voué à la décomposition. Il représentait en "effigie», l'immortalité du corps politique, de la dignité royale conçue comme une institution douée de pérennité. 20

Mais bien avant la Renaissance, l'Église chrétienne s'était ré-approprié le discours originaire de la peinture, qui devait dorénavant servir à la propagation de la foi. On trouve trace, entre autres, de l'empreinte de cette re-prise originaire dans le Trattato de Lomazzo:

Il faut [...] rendre sans cesse des grâces particulières au Christ notre Seigneur, qui voulut lui-même être peintre en imprimant sa très sainte effigie sur le voile de sainte Véronique, afin que restât à la postérité un exemple unique de lui, qui nous inclinât à l'aimer et à le révérer en voyant cette image, comme on le fait à Rome. 21

Et Lomazzo d'ajouter: "Après le Christ, nous avons à révérer l'évangéliste saint Luc, qui nous a laissé, de sa main, le portrait de la Vierge Marie, avec son Fils dans les bras»22.

Ainsi, le portrait, par sa matérialité référentielle mimétique - qui est au cœur de toute la problématique théorique du portrait, soit celle de la ressemblance - et son pouvoir évocateur, qu'il soit amoureux, politique ou religieux, s'inscrit très tôt dans la littérature artistique comme un véhicule idéel. 
Il semble que seule a été retenue comme centrale par ces théoriciens la problématique platonicienne de la ressemblance, soit celle de l'adéquation de la copie par rapport à l'original. L'artiste, pour être l'égal de Dieu, devait minimiser cette différence entre l'original et la copie pour que l'Art puisse égaler la Nature.

Les spécialistes ont toutefois occulté l'analyse de l'élément spatial déployé entre le spectateur-voyeur et l'objet-portrait, qui lui aussi pourrait avoir une incidence sur la capacité d'idéalisation du portrait. Une diminution de ce facteur spatial pourrait entraîner, entre autres, une augmentation de la fréquence de "consultation" du portrait favorisée ainsi par la portabilité de l'objet-portrait et encourager l'accessibilité du spectateur-voyeur au facteur idéel véhiculé par le portrait. Il semble plus approprié de parler ici de "consultation" plutôt que de «contemplation», la portabilité entrainnant, à mon avis, un rapport de nature privée plutôt que publique. L'on peut d'ailleurs lire dans L'Âge d'or du petit portrait (1995) que

[...] plutôt que la contemplation, la miniature appelle l'intimité de la «consultation» Elle est un espace privé, my privacy, diraiton en anglais, dans lequel il faut pénétrer, dans lequel il faut s'absorber, plutôt qu'il ne vient au-devant de vous. ${ }^{23}$

André Thevet ${ }^{24}$, qui réalisa au XVIe siècle le premier «livre-galerie» français d'hommes illustres, relevait, en ces termes, dans ses Portraits et vies des hommes illustres l'espèce de fétichisme qu'on attachait déjà aux portraits et son effet d'entraînement sur la portabilité:

De jour à autre, on voit pratiquer que volontiers nous portons aux anneaux, faveurs et pendants l'image de celui que plus nous aimons ou avons aimé durant leur vie. Et encore ceux desquels [sic] avons reçu quelque présent et bienfait, obligent tant leurs clients à leur effigier simulacres qu'ils les adorent comme dieux. ${ }^{25}$

On voit donc ici se profiler les desseins fétichistes de la miniature. Celui que l'on appelle le «petit portrait» atteignit son apogée au XVIIIe siècle avant de disparaitre lors de l'avènement de la photographie.
C'est bien Walter Benjamin qui disaitdans sa «Petite histoire de la photographie» (1931):

Dès l'instant que Daguerre eut la chance de pouvoir fixer les images de la chambre noire, les peintres, sur ce point, furent congédiés par le technicien. Mais la véritable victime de la photographie ne fut pas la peinture de paysage, ce fut le portrait en miniature [c'est moi qui souligne]. Les choses allèrent si vite que, dès 1840, la plupart des innombrables miniaturistes étaient devenus photographes professionnels, d'abord accessoirement, ensuite de façon exclusive... 26

Avec le portrait en miniature, la peinture avait donc déjà pressenti la nécessité de la portabilité du portrait et ce n'est certes pas un hasard si l'emprise du photographique fit d'abord ses ravages auprès de la miniature.

\section{LA PHOTOGRAPHIE}

Autant le portrait est un cas de figuration pour l'origine de la peinture, autant celui-ci "personnifie» les débuts du photographique. En effet, le premier daguerréotype connu à ce jour ${ }^{27}$, et ce, contrairement à ce que l'on croyait jusqu'à tout récemment ${ }^{28}$, est un portrait, qui plus est le portrait d'un peintre naturaliste, Nicolas Huet, comme si la photographie avait décidé de ce geste parricide et avait dressé dans sa visée, dans sa mire, une effigie in executio; ce portrait fut réalisé en 1837 par Louis-Jacques-Mandé Daguerre (1787-1851), celui que François Arago a gratifié du titre d'inventeur de la photographie lors de la présentation de son rapport sur le daguerréotype à la Chambre des Députés et à l'Académie des Sciences en 183929.

André Gunthert ${ }^{30}$ démontrait, à partir de la correspondance de l'inventeur, son obsession pour la promptitude qui, une fois atteinte, lui permettrait de fixer le premier sur la plaque cette fameuse «image de l'homme" et de pouvoir ainsi en exploiter les bénéfices. Cette recherche de la promptitude, du moindre coût et de la portabilité du matériel photographique tout en s'assurant d'un rendu formel clair et précis ont également incité d'autres personnages, tel William Henry Fox Talbot (18001877), à développer le négatif sur papier et le 
processus du négatif/positif ${ }^{31}$, c'est-à-dire la portabilité et la reproductibilité de l'image. André Adolphe Eugène Disdéri (1819-1890) ${ }^{32}$, de son côté, a inventé le format carte-de-visite, cet archétype de la carte d'affaires... et Roland Barthes ne nous a-t-il pas donné la Chambre Claire à partir d'une réflexion élaborée autour du portrait de sa mère?

Le portrait porte donc non seulement en lui la portabilité, il offre en son sein toute la conjugaison au fil du temps des différents supports médiatiques. Associé au moment originaire, qu'il soit mythique ou non, du pictural et du photographique, il se présente également comme un véhicule idéel. Par sa structure ontologique d'objet-fétiche, il présentéifie l'absence, il réduit ainsi la «distance temporelle» entre le référent et le spectateur-voyeur. Le portrait photographique «portatif» permet une diminution de la «distance spatiale» installée entre le spectateur-voyeur et l'objetportrait. On retrouve donc ici, avec la portabilité du portrait, la combinaison de la disparition ou de la tendance asymptotique de l'évanescence de ces deux éléments qui définissent le monde sensible, soit le temps et l'espace. Il y a lieu de se questionner sur la matérialité et sur cette tendance déjà engagée vers un monde idéel.

Cette proposition de re-lecture du point de rencontre intermédial engagé au XIX ${ }^{\mathrm{e}}$ siècle entre la peinture et la photographie re-hausse ainsi l'importance du portrait comme genre pictural... Félibien doit bien se retourner dans sa tombe! Le portrait est ici passé à l'avant-plan comme un concept permettant d'introduire la portabilité comme ce lieu de rencontre intermédiatique. Cette mélancolie de l'amant, cette blessure narcissique subie par la peinture s'est ici transformée sous l'impulsion de l'amour, du "génie moderne " 33 , en une invention, toute mécanique soit-elle - qui a permis à la peinture de transférer son pouvoir d'évocation amoureux à la photographie pour mieux se remettre en question et se "prendre elle-même en main". La main de l'artiste s'est mécanisée, certes, mais la silhouette dessinée de l'amant sur le mur a peut-être retrouvé son dessein indiciel. Ainsi, d'une approche platonicienne du portrait, c'est-à-dire de l'analyse de cet écart entre la copie et l'original, qui semble au cour de la littérature, nous glissons donc ici davantage vers un regard d'analyse différentielle porté sur le portrait pictural et le portrait photographique sans égard à la mimésis... un peu plus deleuzien peut-être...

\section{NO TES}

1. Le Petit Larousse illustré 1992, Paris, Larousse, 1992.

2. E. Baumgartner et P. Ménard, 1996.

3. A. Ernout et A. Meillet, 1951.

4. Hachette. Le Dictionnaire du français, 1992.

5. J. Picoche, 1992.

6. Hachette. Le Dictionnaire du français, 1992.

7. A. Bosse, cité d'après P. Ortwin Rave, dans Reallexikon zur deutschen Kunstgeschichtem, et repris dans N. Schneider, 1994: 10.

8. É. Pommier, 1998: 18.

9. Ibid.

10. D'aucuns pourraient souligner ici la congruence dans ce discours plus romancé de Pline l'Ancien de l'origine de la peinture et de celle de la sculpture. Il faudrait toutefois porter à leur attention la récupération que l'histoire de l'art a faite au cours du temps de ce texte en y évacuant toute référence originaire à la sculpture pour ne conserver que le performatif pictural. À titre d'exemples, il suffit de penser à la version de Quintilien pour lequel «c'est bien l'ombre portée d'un corps éclairé par le soleil [...] qui déclenche l'invention ", ou à Vasari qui, dans le prologue des Vies, se réfère à Pline l'Ancien, dans une version qui remplace la jeune fille par un Lysien, Gigès: «Se tenant près d'un foyer, il regardait son ombre projetée sur le mur et, soudain, avec un morceau de charbon, il en fixa le contour sur le mur $\%$. On retrouve le même type de récit mythique chez A. Félibien (1666) et on peut lire ce passage dans le poème La Peinture de C. Perreault:

Encore s'il me restait de ce charmant visage

Quelque trait imparfait, quelque légère image,

Ce départ odieux, disait-elle en son cœur,

Quelque cruel qu'il soit, aurait moins de rigueur.

Sur la face du mur marqué de cette trace,

Chacun du beau berger connut l'air et la grâce,

Et l'effet merveilleux de cet événement

Fut d'un art si divin l'heureux commencement.

(É. Pommier, 1998: 19-20)

Nombre d'autres textes pourraient s'ajouter à cette liste et ainsi contribuer à témoigner de l'évacuation de l'origine de la sculpture dans la reprise du mythe développé par Pline l'Ancien.

11. A. Félibien, Entretiens sur les vies et les ouvrages des plus excellents peintres anciens et modernes, Entretien I [1666]; d'après É. Pommier, 1998: 19 .

12. Pour plus de détails sur la théorie freudienne du fétichisme, voir S. Freud, 1987: 62-65; O. Rank, 1988: 147-166 et H. Rey-Flaud, 1994.

13. Pour plus de détails sur la théorie lacanienne de l'objet-fétiche, voir J. Lacan, 1994: 151-179.

14. Pour rester dans l'esprit du fétichisme, on ne peut passer sous silence le livre écrit par G. de Gaeton de Clérambault, 1991.

15. G. B. Marino, La Galeria [Venise, 1619-1620], t. I; d'après É. Pommier, 1998: 185.

16. En effet, Des Frans et Sylvie, à la veille du départ de celui-ci pour 
Rome, échangent leurs portraits. Mais Des Frans se fait attaquer par des brigands lors de son passage dans les Alpes. Il retrouvera le portrait peu de temps après à Grenoble dans la boutique d'un marchand: «Cette vue me rappela tout l'amour que j'avais eu pour elle, je tombai en faiblesse [...] Je le baisai, les larmes aux yeux. Ce marchand sachant qu'il m'avait été volé, me le donna pour ce que je voulus. Je l'emportai et l'ai toujours conservé depuis. Ce portrait affermit la résolution que j'avais prise de retourner vers elle; et je n'eus plus d'autre impatience que celle de monter à cheval». R. Challe, Les Illustres Françaises, 1713, cité dans É. Pommier, 1998: 265.

17. Alberti, Livre II, § 25, 1992: 131.

18. D’après É. Pommier, 1998: 456

19. Kantorowicz, Les Deux Corps du roi. Essai sur la théologie politique du Moyen Âge, d'après J. von Schlosser, [1911] 1997: 194.

20. Schlosser, [1911] 1997: 198.

21. G. P. Lomazzo, Trattato dell'arte della pittura, scoltura ed architectura [Milan, 1584]; d'après É. Pommier, 1998: 159.

22. Ibid.

23. L'Âge d'or du petit portrait (catalogue d'exposition), 1995: 10.

24. A. Thevet a écrit le livre Portraits et vies des hommes illustres présenté comme une galerie imaginaire des portraits des hommes illustres. On est donc passé avec Thevet de la galerie réelle de portraits comme celle qu'avait réalisée Paolo Giovio (1540), humaniste, philosophe, médecin et historien dans sa villa, conçue comme un temple de la vertu, appelée Museo et consacrée au plaisir du public au livre-galerie, grâce à l'imprimerie et à la gravure. Voici donc un autre exemple de la portabilité favorisée par l'avènement de ces médias.

25. A. Thevet, d'après É. Pommier, 1998: 197.

26. W. Benjamin, [1931] 1971: 22.

27. Le daguerréotype (1837) du peintre naturaliste Nicolas Huet a été découvert dans un marché aux puces par Marc Pagneux, marchand d'art et expert, selon un article publié dans Libération, 33, lundi 2 nov. 1998. 28. Vue du boulevard du Temple (1839), daguer réotype réalisé par LouisJacques-Mandé Daguerre, était considéré jusqu'à tout récemment comme le plus ancien de l'histoire de la photographie.

29. Pour plus de détails sur la présentation de F. Arago, voir «François Arago, 1839. Rapport sur le daguerréotype" dans M. Frizot et

F. Ducros, 1987: 10-14.

30. A. Gunthert, 1998: 4-25.

31. Pour plus de détails, voir N. Rosemblun, 1989: 24-32 et 54-56.

32. Voir "Eugène Disderi, 1862. Sur le portrait photographique», dans M. Frizot et F. Ducros, 1987 : 37-48.

33. Il est fait référence ici à la double articulation (mélancolie poétique et melancholia generosa) du concept de mélancolie développée par Aristote dans le Problème XXX.I et reprise par Marsile Ficin à la Renaissance. La mélancolie prit en effet avec l'humanisme italien une "valeur de force intellectuelle positive». C'est d'ailleurs l'utilisation de ce concept de mélancolie qui a permis de développer cette nouvelle approche de la rencontre intermédiale survenue au XIX siècle entre le pictural et le photographique. «Élever la mélancolie au rang de force intellectuelle, c'était évidemment bien autre chose que de l'interpréter comme un état émotif et subjectif. Les deux tendances peuvent se combiner, dans la mesure où la valeur émotive de l'humeur sentimentale et voluptueuse peut s'enrichir de la valeur intellectuelle de la mélancolie contemplative ou artistiquement féconde [...]. Ainsi, le système de Ficin - et c'est peut-être là sa vertu la plus grande réussit-il à doter la "contradiction immanente" de Saturne d'un pouvoir rédempteur : l'homme de génie mélancolique - que sa soumission à Saturne faisait souffrir, dans la mesure où l'astre infligeait à son corps et à ses facultés inférieures tristesse, peur et abattement - pouvait trouver salut dans l'acte délibéré de se tourner vers Saturne. Autrement dit, le mélancolique devait, de son propre mouvement, s'appliquer à cette activité qui constitue le domaine spécifique de l'astre sublime de la méditation, et la planète favorise avec une ardeur égale à celle avec laquelle elle fait obstacle et nuit aux fonctions ordinaires du corps et de l'âme: la contemplation créatrice, qui a son siège dans la "mens" et nulle part ailleurs. En tant qu'ennemi et oppresseur de toute vie qui est, en quelque manière, assujettie au monde présent, Saturne engendre la mélancolie; mais en tant qu'ami et protecteur d'une existence supérieure et purement intellectuelle, il peut aussi la guérir ». R. Klibansky, E. Panofsky et F. Saxl, 1989: 389-390, 427.

\section{RÉFÉREN CES BIBLIO GRAPHIQ U ES}

AlberTI [1992]: De la peinture. De Pictura (1435), trad. par J.L. Schefer, Paris, Macula Dedale.

BARTHES, R. [1980] : La Chambre claire. Note sur la photographie, Paris, Gallimard/Seuil.

BAumgaRTNER, E. et P. MÉNARD [1996]: Dictionnaire étymologique et historique de la langue française, Paris, Librairie Générale Française, coll. "Les Usuels de Poche».

Benjamin, W. [1931]: «Petite histoire de la photographie», dans Euvres II. Poésie et Révolution, Paris, Denoël, coll. «Dossiers des Lettres Nouvelles ", 1971, 15-35.

ERnout, A. et A. MEILlet [1951]: Dictionnaire étymologique de la langue latine. Histoire de mots, $3^{e}$ éd., Paris, Librairie C. Klincksieck.

FREUD, S. [1987]: «Substitut impropre de l'objet sexuel - Fétichisme», dans Trois Essais sur la théorie sexuelle, Paris, Gallimard, coll. «Folio essais ", 62-65.

FrIzOT, M. et F. Ducros (dir.) [1987]: Du bon usage de la photographie. Une anthologie de textes, Paris, Centre National de la Photographie. Gaeton de Clérambault, G. de [1991]: Passion érotique des étoffes chez la femme, Paris, coll. «Les empêcheurs de penser en rond".

GUNTHERT, A. [1998]: « Daguerre ou la promptitude. Archéologie de la réduction du temps de pose", dans Études photographiques, n ${ }^{\circ}$, novembre.

Hachette. Le Dictionnaire du français, Paris, Hachette, 1992.

KLIBANSky, R., E. PANOFSKY et F. SAXL [1964]: Saturne et la mélancolie, trad. par F. Durand-Bogaert et L. Évrard, Paris, Gallimard, 1989.

LACAN, J. [1956-1957]: Le Séminaire. Livre IV: La relation d'objet, Paris, Seuil, 1994, 151-179.

L'Âge d'or du petit portrait [1995]: catalogue d'exposition [exposition itinérante Bordeaux, Genève et Paris], Paris, Réunion des Musées nationaux.

Madame de La Fayette [1678] : La Princesse de Clèves, Paris, GFFlammarion, 1966.

PICOCHE, J. [1992]: Dictionnaire étymologique du français, Paris,

Dictionnaire Le Robert.

POMmier, É. [1998]: Théories du portrait. De la Renaissance aux Lumières, Paris, Gallimard.

RANK, O. [1988] : «Freud and Fetishism : Previously Unpublished Minutes of the Vienna Psychoanalytic Society", Psychoanalytic Quaterly, LVII, 147-166.

REY-FLAUD, H. [1994] : Comment Freud inventa le fétichisme... et réinventa la psychanalyse, Paris, Payot et Rivages.

Rosemblun, N. [1989]: A World History of Photography, 2e éd., New York, Abbeville Press Publishers.

SCHLOSSER, J. von [1911]: Histoire du portrait en cire, trad. de l'allemand par É. Pommier, Paris, Macula, 1997.

SCHNEIDER, N. [1994]: L'Art du portrait, Cologne, Taschen. 